\title{
Quantification of Posture-Induced Changes in Bed-Based Ballistocardiogram
}

\author{
Hewon Jung ${ }^{1}$, Jacob Kimball ${ }^{1}$, Timothy Receveur ${ }^{2}$, Eric Agdeppa ${ }^{2}$, Omer T Inan ${ }^{1}$ \\ ${ }^{1}$ School of Electrical and Computer Engineering, Georgia Institute of Technology, Atlanta, GA, USA \\ ${ }^{2}$ Hill-Rom, Chicago, IL, USA
}

\begin{abstract}
The ballistocardiogram (BCG), a measurement of cardiogenic whole body movements, is a technique that enables non-invasive cardiovascular monitoring. A main challenge of the BCG signal is that its morphology and amplitude are sensitive to the posture of the subject during the recording period.

This work elucidates effects of posture on bed-based $B C G$ recordings by (1) creating templates for standing $B C G$ signals obtained from subjects in a prior study, and (2) quantifying the distance between these templates and $B C G$ waveforms obtained in different body postures on the bed for a new set of subjects. The signal quality index (SQI), defined in previous work and corresponding to the inverse of the distance to the templates, was the highest for the supine posture and the lowest for the lateral postures.

A previously-reported system identification approach to correct for distortions in the lateral, prone, and seated postures was further validated. The system identification algorithm significantly improved the signal quality and correlation to the reference morphology - the supine and standing BCG. This work has implications for robust signal processing that allows for accurate physiological interpretation of the BCG obtained in a variety of postures from a subject in bed.
\end{abstract}

\section{Introduction}

Continuous and unobtrusive vitals monitoring has gained attention for the treatment and prevention of diseases as the number of patients with chronic cardiorespiratory conditions grows. In home settings, such monitoring may allow longitudinal tracking of the patient's physiological conditions $[1,2]$. Furthermore, it serves a key role in hospitals for patient safety and earlier detection of patient deterioration without increasing the burden on caregivers [3].

Advances in sensing cardiogenic vibration signals have paved the way for unobtrusive vitals monitoring. One of the most commonly investigated sensing modalities for unobtrusive monitoring is the ballistocardiogram (BCG) [4]. The BCG measures the microdisplacement of the whole body in response to the movement of blood caused by cardiac ejection [5]. Recent literature has reported promising results for BCG use on monitoring physiological indicators with unobtrusive, everyday objects such as a bed or a bathroom scale, which do not interfere with normal daily activities [6,7].

However, BCG measurement systems are sensitive to the posture of the subject during the recording period, in that the signal shape may be distorted when the subject's posture changes [8]. For BCG signals measured with a weighing scale, subjects are required to stand upright and still to obtain high-quality signals. Any modification in the posture of the subject such as slouching will distort signal morphology, making the physiological interpretation of the BCG challenging. Additionally, for bed-based BCG recordings, commonly used for long-term monitoring such as overnight sleep studies [9], changes in body posture are inevitable - subjects may lay supine for part of the night, then laterally for some time, then prone, for example. Moreover, bed-based BCG is even more susceptible to postural effects than standing BCG as the head-to-foot and dorso-ventral forces are unavoidably coupled together in the measurement [4].

To investigate postural influence on BCG signals, the postural effects on standing and seated BCG signals measured with the scale have been quantified and corrected in [8]. However, such an approach has never been used for bed-based BCG. This work quantifies the effects of body posture on the bed on BCG signals measured with the load cells in a hospital patient bed. The quality of BCG signals from five different postures - supine, left / right lateral, prone, and seated are demonstrated using the standing BCG as a reference. Signal quality was assessed with a previously described method in [10]. Furthermore, signal morphology was corrected with the system identification method found in [8].

\section{Methods}

\subsection{Experimental Protocol}

In total, 11 healthy subjects (Male: 6, Female: 5; Age: $27.8 \pm 4$; Weight: $71.54 \pm 19.5 \mathrm{~kg}$; Height: $172.27 \pm 12.3$ 

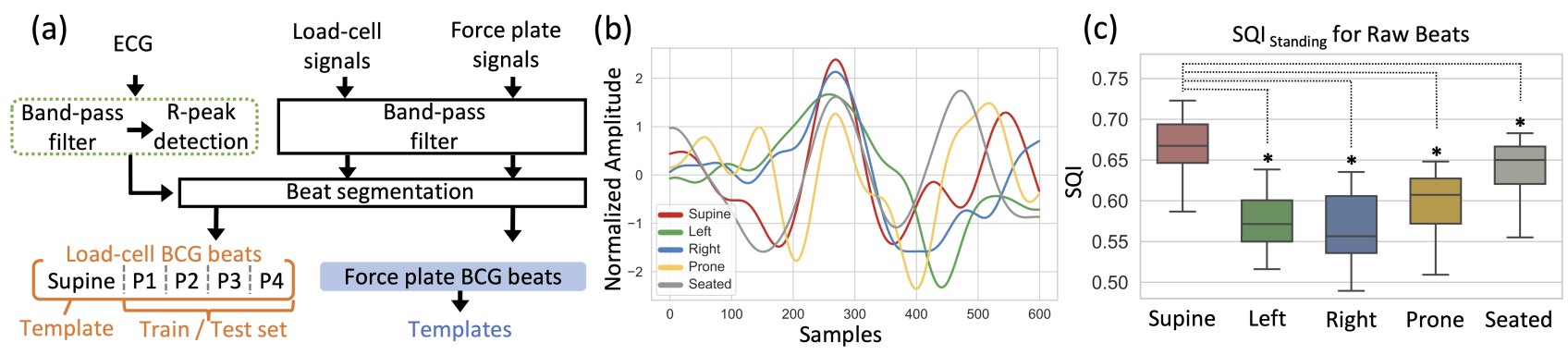

Figure 1. (a): A block diagram of pre-processing steps. (b): An example plot of ensemble-averaged raw beats for each posture. (c): A boxplot of SQIs computed over standing BCG templates. Ensemble-averaged raw beats were used for each posture to compute the SQI. * indicates a significant difference between each posture and the supine posture with a $95 \%$ confidence level.

$\mathrm{cm})$ were recruited for this study conducted under the approval of the Georgia Institute of Technology Institutional Review Board (IRB).

BCG signals from the load cells embedded in a patient bed (Centrella ${ }^{\mathrm{TM}}$, Hill-Rom) along with the reference Lead II electrocardiogram (ECG) signal were recorded from a Biopac BN-ECG2 system while subjects were on the bed. The outputs from the load cells were amplified through a custom-designed circuit similar to [11]. All signals were continuously recorded via a data acquisition unit (DAQ, MP150, Biopac Systems) with a sampling rate of 1 $\mathrm{kHz}$.

During the protocol, subjects were asked to lay down on the bed and remain still in five different postures including supine $\left(P_{0}\right)$, left and right lateral $\left(P_{1}\right.$ and $\left.P_{2}\right)$, prone $\left(P_{3}\right)$, and seated $\left(P_{4}\right)$. The seated posture required the bed to be adjusted to the seated configuration. Subjects remained in each posture for one minute.

To generate templates of the BCG signal measured in the standing upright posture, the data set from another study was used. This data set consists of 30 healthy patients (Male: 20, Female: 10, Age: $26.4 \pm 3.2$, Weight: $70.95 \pm$ $14.43 \mathrm{~kg}$; Height: $172.94 \pm 9.69 \mathrm{~cm}$ ). The subjects were asked to stand upright on the force plate for two minutes while the BCG and ECG signals were recorded.

\subsection{Pre-Processing}

A block diagram of pre-processing steps is shown in Figure 1(a). All signals were band-pass filtered with cutoff frequencies of $1-40 \mathrm{~Hz}$ for ECG and $0.5-12 \mathrm{~Hz}$ for BCG. ECG R-peaks were detected by thresholding and used as a reference for segmenting BCG signals into beats corresponding to cardiac cycles. For each BCG beat, a few samples before the ECG R-peaks were padded to account for the filter length $t$ in the system identification, which will be discussed in Section 2.3. This segmentation resulted in a frame length of $d=600$ samples after R-peaks
+ filter length $t$. The padded segment of length $t$ was removed for testing.

A set of BCG beats extracted from the recordings while the $k^{\text {th }}$ subject was in the supine posture - denoted as $B_{s}^{k}$ - was used to generate a template for the supine posture. Templates of supine $\left(\boldsymbol{t}_{f}\right)$ and standing $\left(\boldsymbol{t}_{s}^{k}\right)$ BCG signals were generated as done in [10], and the set of standing BCG templates from all $N=30$ subjects was defined as $T_{f}=\left\{\boldsymbol{t}_{f}^{1}, \boldsymbol{t}_{f}^{1}, \ldots, \boldsymbol{t}_{f}^{N}\right\}$.

For all other postures, beats from the first 20 seconds of the one-minute recording were smoothed with an exponential moving average filter with a window of five beats, and included in a training set $B_{i}^{k}\left(B_{i}^{k} \in \mathbb{R}^{(+\approx) \times \mathbb{M}}, i=\right.$ posture $P_{i}$, $M=$ number of heartbeats). The remaining beats from each posture were included in a testing set $U_{i}^{k}\left(U_{i}^{k} \in \mathbb{R}^{\lessdot \times \mathbb{M}^{\prime}}, i\right.$ $=$ posture $P_{i}, l=600, M^{\prime}=$ number of heartbeats). For each training and testing set, beats from left and right foot channels were included in equal proportions.

\subsection{BCG Morphology Correction}

To correct the BCG morphology distorted by the posture, a transformation function that maps distorted BCG signals to "good-posture" BCG signals was found. In this study, BCG signals from the supine posture were considered as "good-posture" BCG signals for beat correction, as the signal quality was the highest in the supine posture based on our analysis in Section 3, and prior knowledge of BCG signals [4]. This transformation function can be determined via a linear system identification method [8], which can be formulated as a least-squares problem.

Here, for each subject $k$ and posture $P_{i}$, the matrix $A$ was composed with lagged vectors from each training beat $\boldsymbol{b}_{i, j}^{k}$ ( $\boldsymbol{b}_{i, j}^{k} \in B_{i}^{k}, i=$ posture $P_{i}, j=j^{\text {th }}$ beat), and the output vector $y$ was made up with samples from the supine template $\boldsymbol{t}_{s}^{k}$. The variable $x$ serves as 1-D FIR filter coefficients for a linear mapping between each training beat and the supine BCG template. With the filter order $t$, and $H_{i}^{k}$ denoting a 
transformation from posture $P_{i}$ to the supine template $\boldsymbol{t}_{\boldsymbol{s}}^{k}$, mathematical expressions of $y, A$, and $x$ are given as:

$$
\begin{aligned}
y & =\left[\begin{array}{llll}
\boldsymbol{t}_{s}^{k}[t] & \boldsymbol{t}_{s}^{k}[t+1] & \ldots & \boldsymbol{t}_{s}^{k}[d]
\end{array}\right]^{T} \\
x & =\left[\begin{array}{llll}
H_{i}^{k}[1] & H_{i}^{k}[2] & \ldots & H_{i}^{k}[t]
\end{array}\right]^{T} \\
A & =\left[\begin{array}{cccc}
\boldsymbol{b}_{i, j}^{k}[t] & \boldsymbol{b}_{i, j}^{k}[t-1] & \ldots & \boldsymbol{b}_{i, j}^{k}[1] \\
\boldsymbol{b}_{i, j}^{k}[t+1] & \boldsymbol{b}_{i, j}^{k}[t] & \ldots & \boldsymbol{b}_{i, j}^{k}[2] \\
\vdots & \vdots & \ddots & \vdots \\
\boldsymbol{b}_{i, j}^{k}[d] & \boldsymbol{b}_{i, j}^{k}[d-1] & \ldots & \boldsymbol{b}_{i, j}^{k}[d-t+1]
\end{array}\right]
\end{aligned}
$$

The filter length $t$ was determined through 3-fold cross validation and used for the final training. The least-squares solution $\hat{\mathbf{x}}$ that minimizes the $l_{2}$-norm of error in (4) can be expressed as (5). The Tikhonov regularization term $\delta$ was added in (4) as done in [8], and was set to 0.01 .

$$
\begin{array}{r}
\min _{x \in \mathbb{R}^{\mathbb{M}}}\|y-A x\|_{2}^{2}+\delta\|x\|_{2}^{2} \\
\hat{\mathbf{x}}=\left(A^{T} A+\delta I\right)^{-1} A^{T} y
\end{array}
$$

The FIR coefficients $\hat{\mathbf{x}}$ found for each training beat were averaged across all training beats of posture $P_{i}$, and the averaged coefficients were used as the transformation function $H_{i}^{k}$ during testing.

\subsection{Evaluation}

The evaluation of this work was based on the signal quality index (SQI), a function of the inverse distance between a captured signal and the reference template. There are multiple methods to estimate distance, but the dynamic-time feature matching (DTFM) distance in [10] was used in this study. The work in [10] has shown this metric to be a reliable measure of the signal quality for noninvasive cardiac bio-signals.

To compare the morphology of raw beats and the corrected beats for each posture, the SQI and Pearson correlation coefficient were calculated. The three metrics computed for each testing beat $\boldsymbol{u}_{i, j}^{k}\left(\boldsymbol{u}_{i, j}^{k} \in U_{i}^{k}\right)$ were SQI against the standing BCG templates ( $S Q I_{\text {standing }}$ ), SQI against the supine templates $\left(S Q I_{\text {supine }}\right)$, and correlation to the supine template $\left(\rho_{\text {supine }}\right)$. Note that $S Q I_{\text {supine }}$ and $\rho_{\text {supine }}$ are for intra-subject evaluation, such that scores were computed against the supine template of the subject corresponding to the test beats. SQIs were averaged over all testing beats of each posture and the mean SQIs were reported as the final scores. For consistent scaling, corrected, raw, and template beats were normalized for SQI computation.

\section{Results and Discussion}

Figure 1(b) shows an example plot of ensembleaveraged raw beats for each posture. Note that the whole protocol was 10-minutes long without any interventions that could result in physiological changes. Therefore, the differences in morphology between postures shown in Figure 1(b) are due mainly to postural changes. The $S Q I_{\text {standing }}$ shown in Figure 1(c) quantifies this observation. The median SQI for the supine posture was the highest with 0.67 , followed by the seated posture $(0.65)$. Left and right lateral postures showed the lowest scores ( 0.57 and 0.56 , respectively), while the prone posture was slightly higher with a score of 0.60 . The SQIs here were computed with the whole interval, which includes both the training and testing beats for each posture. The seated posture in this work had relatively high SQI unlike the previous study [8], where significant distortions were observed. This could possibly be due to the difference in the method of measurement - in this work, the load-cells were fixed to the frame of the patient bed, while the scale was placed between the subject and the chair in [8], and thus the dampened BCG forces were directly reflected in the recordings.

The supine posture had significantly higher $S Q I_{\text {standing }}$ than all other postures, indicating that the morphology in this posture - among the five postures explored - is closest to the BCG measured in the standing upright posture, a reference standard for the BCG measurement. Here, the standing BCG templates are only used as the reference for the evaluation of signal quality. For the beat correction, BCG recordings of each subject in supine posture the posture with the highest signal quality - were used as the reference. This is because the standing BCG templates were measured from other subjects or at a different time; thus the linear mapping of training beats to standing BCG templates will lose temporal physiological information specific to the subject. Linear mapping to supine BCG templates, on the other hand, would reflect that information as they were measured from the specific subject at the same time point.

Figure 2(a) shows $S Q I_{\text {standing }}$ for both raw and corrected test beats. Corrected beats have significantly higher SQI than the raw beats for all postures. The same trend was observed in Figure 2(b), where the SQI was computed against the supine templates for test beats. The decrease in distance to standing BCG templates demonstrates the improvement in signal quality in general, based on the gold standard measurement. Furthermore, the decreased distance to supine templates indicates that the transformation allows for consistency among BCG beats that are measured at the same time point. The correlation to supine templates in Figure 2(c) also supports this result, showing significant improvement after correction.

\section{Conclusion}

In this work, changes in BCG morphology induced by the body posture on the patient bed were quantified. The 

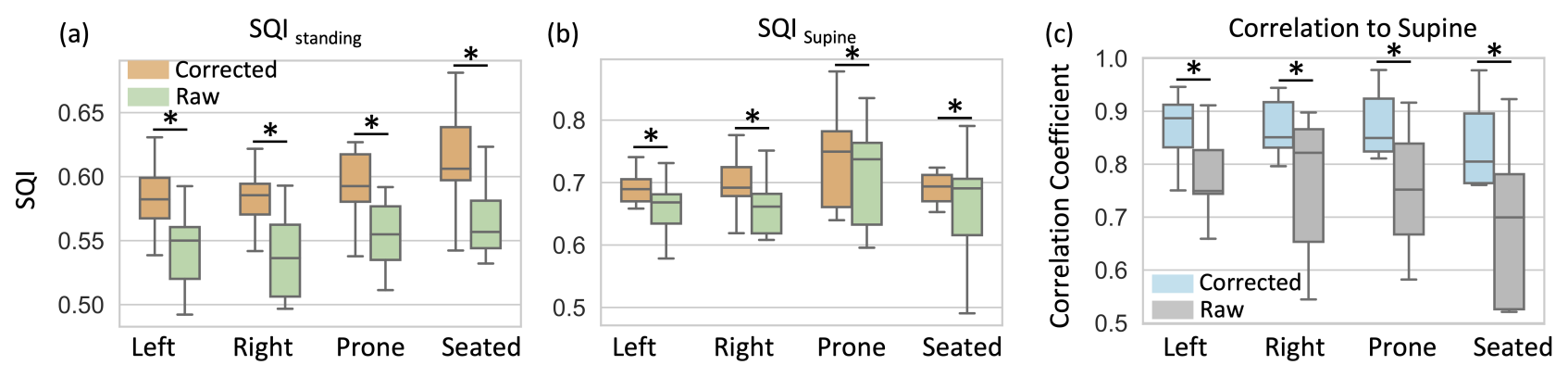

Figure 2. (a)A box plot of $S Q I_{\text {standing }}$ for raw and corrected beats. (b) A box plot of $S Q I_{\text {supine }}$ for raw and corrected beats. (c) Pearson correlation coefficient between each posture and the supine template, before and after correction. * indicates a significant difference between the corrected and raw beats for each posture with $95 \%$ confidence level.

assessment of signal quality was done - for the first time - based on the distance between bed-based BCG and the standard standing BCG. Furthermore, system identification demonstrably corrected distortions in the signal morphology due to different body postures.

Correcting postural effects on signal morphology may not be required for the estimation of cardiac cycles as posture distortion would consistently appear in the same pattern across neighboring beats while the posture is maintained. However, removing these effects is important for investigating the in-depth correlation of BCG signals to cardiorespiratory parameters, particularly those related to timings and amplitudes of the I-, J-, and K-points of the $\mathrm{BCG}$, such as pre-ejection period (PEP), pulse transit time, and aortic pulse amplitude [5]. The effects of morphology correction on estimating such cardiorespiratory parameters could be done as future work.

\section{Funding and Disclosures}

This project was funded by a research contract from Hill-Rom Services, Inc. to the Inan Research Lab at Georgia Institute of Technology. O. T. Inan is a Scientific Advisor for Physiowave, Inc., has research funding for his lab from Hill-Rom Services, Inc. and Murata Americas, and has IP licensed by Physiowave and TandemLaunch.

\section{References}

[1] Ziegler PD, Koehler JL, Mehra R. Comparison of continuous versus intermittent monitoring of atrial arrhythmias. Heart Rhythm 2006;ISSN 15475271.

[2] Konstam MA. Home monitoring should be the central element in an effective program of heart failure disease management. Circulation 2012;ISSN 00097322.

[3] Cuthbertson BH, Boroujerdi M, McKie L, Aucott L, Prescott G. Can physiological variables and early warning scoring systems allow early recognition of the deteriorating surgical patient? Critical Care Medicine 2007;ISSN 00903493.
[4] Inan OT, Migeotte PF, Park KS, Etemadi M, Tavakolian K, Casanella R, Zanetti J, Tank J, Funtova I, Prisk GK, Di Rienzo M. Ballistocardiography and Seismocardiography: A Review of Recent Advances. IEEE Journal of Biomedical and Health Informatics 2015;ISSN 21682194.

[5] Kim CS, Ober SL, McMurtry MS, Finegan BA, Inan OT, Mukkamala R, Hahn JO. Ballistocardiogram: Mechanism and Potential for Unobtrusive Cardiovascular Health Monitoring. Scientific Reports 2016;ISSN 20452322.

[6] Brüser C, Stadlthanner K, De Waele S, Leonhardt S. Adaptive beat-to-beat heart rate estimation in ballistocardiograms. IEEE Transactions on Information Technology in Biomedicine 2011; ISSN 10897771.

[7] Carek AM, Inan OT. Robust sensing of distal pulse waveforms on a modified weighing scale for ubiquitous pulse transit time measurement. IEEE Transactions on Biomedical Circuits and Systems 2017;ISSN 19324545.

[8] Javaid AQ, Wiens AD, Fesmire NF, Weitnauer MA, Inan OT. Quantifying and reducing posture-dependent distortion in ballistocardiogram measurements. IEEE Journal of Biomedical and Health Informatics 2015;ISSN 21682194.

[9] Choi BH, Chung GS, Lee JS, Jeong DU, Park KS. Slowwave sleep estimation on a load-cell-installed bed: A nonconstrained method. Physiological Measurement 2009; ISSN 09673334.

[10] Zia JS, Kimball J, Hersek S, Shandhi M, Semiz B, Inan O. A unified framework for quality indexing and classification of seismocardiogram signals. IEEE Journal of Biomedical and Health Informatics 2019;ISSN 2168-2194.

[11] Inan OT, Etemadi M, Paloma A, Giovangrandi L, Kovacs GT. Non-invasive cardiac output trending during exercise recovery on a bathroom-scale-based ballistocardiograph. Physiological Measurement 2009;ISSN 09673334.

Address for correspondence:

Hewon Jung

Georgia Institute of Technology, Atlanta, GA, 30332, USA

hewon.jung@gatech.edu 